\title{
Decreased Tacrolimus Levels after Administration of Rifampin to a Patient with Renal Transplant
}

\author{
Heather Naylor and Jenna Robichaud
}

\section{INTRODUCTION}

$\mathrm{T}$ he calcineurin inhibitor tacrolimus is used to prevent organ rejection following renal transplant. This drug is metabolized through the hepatic cytochrome P450 (CYP450) $3 \mathrm{~A}$ enzymes, in particular, CYP3A4 and CYP3A5.,2 A strong relationship between CYP3A5 genetic polymorphisms and the pharmacokinetics of tacrolimus has been demonstrated in kidney, heart, and liver graft recipients. ${ }^{1,2}$ Previous studies have shown that carriers of the $C Y P 3 A 5^{*} 1$ allele require larger doses of tacrolimus to achieve target blood concentrations than patients with other alleles. ${ }^{3-6}$ The frequency of this allele varies depending on ethnicity and is more common among Asian and African American people than among whites. ${ }^{7}$

Rifampin is known to affect the metabolism of tacrolimus through induction of CYP $3 \mathrm{~A}^{8}$ and, to a far lesser extent, CYP3A5. ${ }^{9}{ }^{10}$ Although the interaction between these drugs is in theory well recognized, its clinical significance in adults has been reported for only 4 Asian patients, ${ }^{11-14} 2$ Hispanic patients, ${ }^{15,16}$ and one Kuwaiti patient. ${ }^{17}$ To our knowledge, this interaction has not been described previously for adult white patients, the population with the lowest frequency of CYP $3 A 5^{*} 1$ allele carriers. Here, we describe a white patient who had undergone a renal transplant and who experienced a clinically significant interaction between tacrolimus and rifampin.

\section{CASE REPORT}

A 65-year-old white woman (height $162.5 \mathrm{~cm}$, weight $86.2 \mathrm{~kg}$ ) with end-stage renal disease secondary to polycystic kidney disease received a renal allograft from a deceased donor in March 2006.* Post-transplant immunosuppression was maintained with tacrolimus (Prograf), mycophenolate sodium (Myfortic), and prednisone. In December 2011 the patient

\footnotetext{
*The patient provided consent for publication of this case.
}

presented with a few days' history of nausea, abdominal pain, and persistent headache. Her medical history was significant for hypertension, hypothyroidism, and a ventriculoperitoneal (VP) shunt, which had been placed surgically in 1985 for hydrocephalus. Abdominal computed tomography (CT) revealed a $4-\mathrm{cm}$ inclusion cyst in close proximity to the VP shunt tube. However, head CT showed that the VP shunt was functioning, with no evidence of hydrocephalus. The patient's headache and abdominal pain diminished without surgical intervention, and she was discharged 3 days later.

Two weeks after discharge, the patient's nausea, abdominal pain, and headache symptoms returned, and she was readmitted to hospital with suspected shunt blockage and possible infection of the cerebrospinal fluid. Vital signs on admission were as follows: blood pressure 154/71 $\mathrm{mm} \mathrm{Hg}$, heart rate $90 / \mathrm{min}$, respiratory rate $18 / \mathrm{min}$, partial pressure of oxygen $96 \%$ (room air), and temperature $37.0^{\circ} \mathrm{C}$. The neck showed no signs of stiffness. The VP shunt valve did not appear to empty well.

Home medications at the time of readmission were enteric-coated acetylsalicylic acid $81 \mathrm{mg} /$ day, ranitidine 300 $\mathrm{mg} /$ day, simvastatin $10 \mathrm{mg} /$ day, zopiclone $5 \mathrm{mg} /$ day when needed, levothyroxine $0.1 \mathrm{mg} /$ day, amlodipine $10 \mathrm{mg} /$ day, alendronate $70 \mathrm{mg}$ every Thursday, prednisone $5 \mathrm{mg} / \mathrm{day}$, mycophenolate sodium $720 \mathrm{mg} /$ day, and tacrolimus $4 \mathrm{mg} /$ day (target trough level 5-8 ng/mL). All home medications were continued throughout the hospital stay. Of note, the patient had completed a 7-day course of oral ampicillin 1 day before the readmission.

The following abnormal laboratory results were pertinent: C-reactive protein $128 \mathrm{mg} / \mathrm{L}$ (normal range $\leq 7.9 \mathrm{mg} / \mathrm{L}$ ), hemoglobin $110 \mathrm{~g} / \mathrm{L}$ (normal range $115-160 \mathrm{~g} / \mathrm{L}$ ), neutrophils $9.1 \times 10^{9} / \mathrm{L}$ (normal range $2.0 \times 10^{\%} / \mathrm{L}$ to $7.0 \times 10^{\%} / \mathrm{L}$ ), leukocytes in the cerebrospinal fluid $935 \times 10^{6} / \mathrm{L}$ (normal range up to $5 \times 10^{6} / \mathrm{L}$ ), protein in the cerebrospinal fluid 
$0.87 \mathrm{~g} / \mathrm{L}$ (normal range $0.15-0.45 \mathrm{~g} / \mathrm{L}$ ), and tacrolimus trough level $11.4 \mathrm{ng} / \mathrm{mL}$.

Fluid from the VP shunt was sent for culture upon suspicion of infection. Empiric therapy with ceftriaxone $2 \mathrm{~g}$ IV daily and rifampin $600 \mathrm{mg} /$ day was started on postadmission day 1 (PAD1), as recommended by the infectious diseases service. The VP shunt fluid subsequently grew Staphylococcus epidermidis susceptible to vancomycin and rifampin, but resistant to penicillin. Vancomycin was therefore added to the patient's therapy on PAD3. Treatment with ceftriaxone and rifampin was continued.

A decrease in tacrolimus levels was apparent after 4 days of antibiotic therapy. This decrease was attributed to rifampin in the absence of literature reports of clinically significant drug interactions between tacrolimus and vancomycin or ceftriaxone. The tacrolimus trough level decreased from $6.1 \mathrm{ng} / \mathrm{mL}$ on PAD5 to $3.7 \mathrm{ng} / \mathrm{L}$ by PAD7. Therapeutic drug monitoring of tacrolimus was performed by a nephrology inpatient clinical pharmacist (J.R. or H.N.) in collaboration with a nephrologist. Over the next 4 days, the dose of tacrolimus was progressively increased, to $9 \mathrm{mg} / \mathrm{day}$, to attain and maintain the target tacrolimus trough level of 5-8 ng/mL (Figure 1). During this period, serum creatinine declined from baseline values of 90-110 $\mathrm{mol} / \mathrm{L}$ (Figure 2). On PAD13 the patient underwent surgery for removal of the existing VP shunt, drainage of shunt remnants, and placement of a new shunt. Triple therapy with ceftriaxone, rifampin, and vancomycin was continued for 7 days after the procedure, with discontinuation on PAD20.

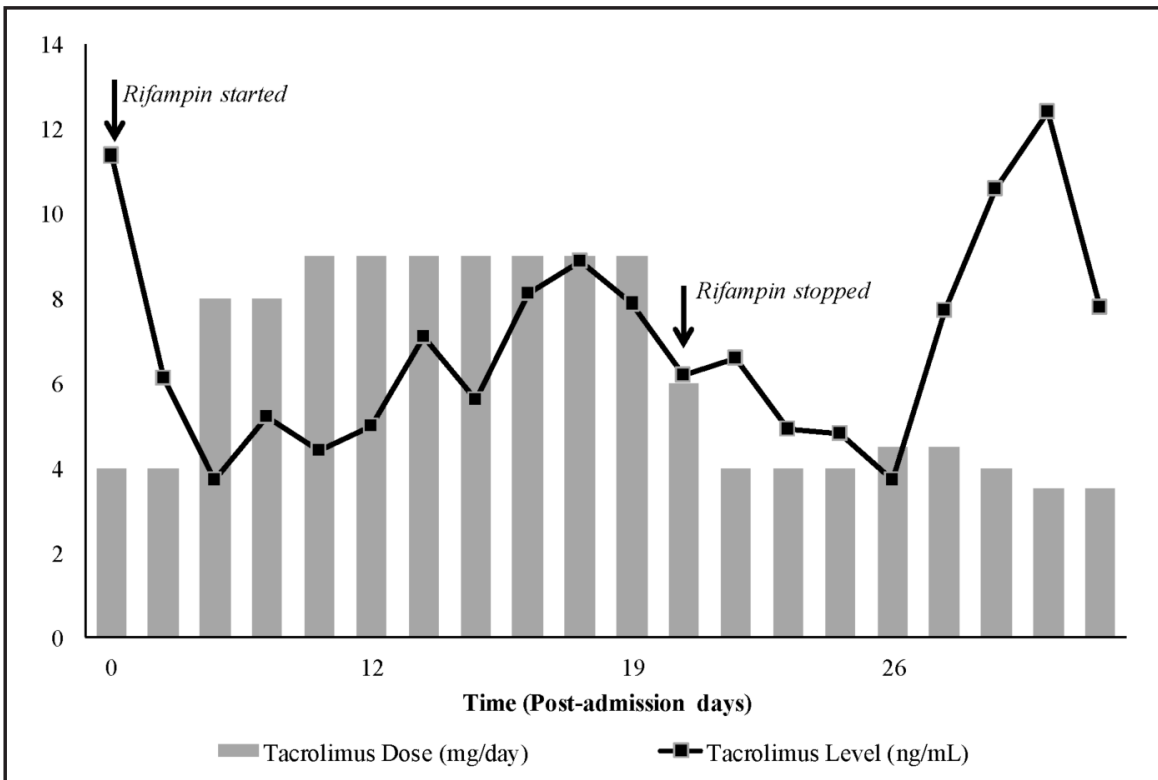

Figure 1. Dose and trough level of tacrolimus during the hospital stay.

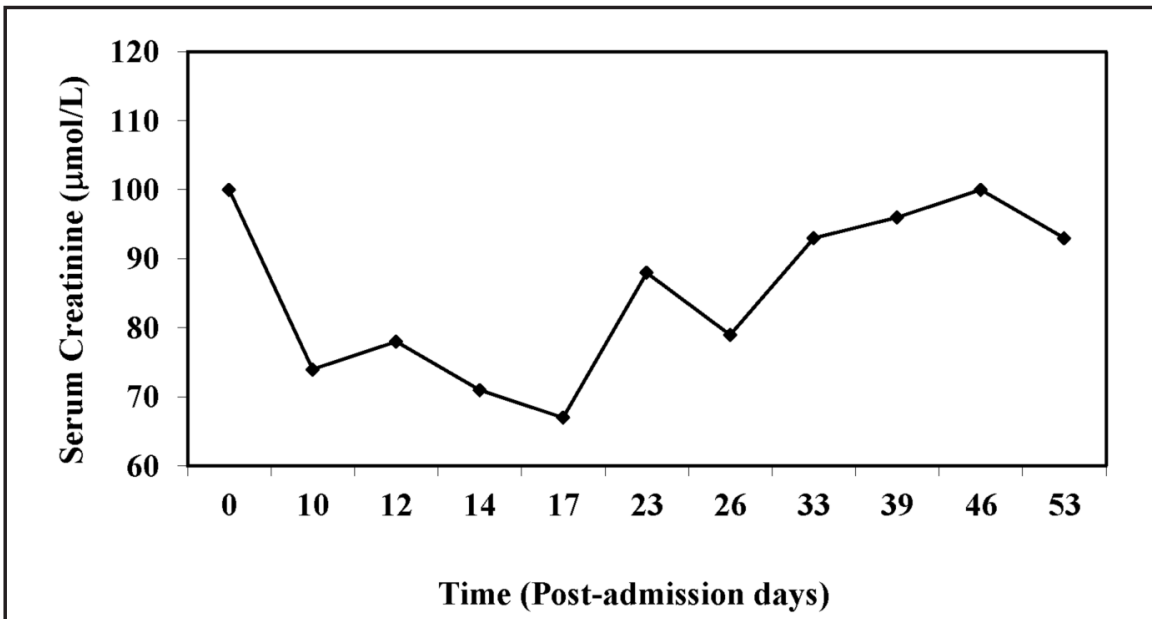

Figure 2. Serum creatinine level during the hospital stay. 
Following discontinuation of rifampin, the dose of tacrolimus was decreased over several days to the patient's preadmission dose of $4 \mathrm{mg} /$ day. However, on PAD26 (6 days after discontinuation of rifampin), the tacrolimus trough level once again declined to $3.7 \mathrm{ng} / \mathrm{mL}$, and an increase in the dose of this drug was required. The patient was discharged from hospital on PAD33. Therapeutic drug monitoring of tacrolimus was continued through an outpatient clinic. The patient's tacrolimus dose was decreased gradually over several weeks to maintain therapeutic levels of this drug. At the time of writing (mid-2013), the patient was continuing to take tacrolimus $3.5 \mathrm{mg} /$ day, graft function was stable, and the patient was clinically well.

\section{DISCUSSION}

To our knowledge, this case is the first report of a clinically significant interaction between tacrolimus and rifampin in an adult white patient. Addition of rifampin to the medication regimen resulted in a reduction in serum tacrolimus concentrations requiring subsequent dose increases to maintain desired therapeutic levels. Furthermore, the impact of rifampin on tacrolimus metabolism was sustained for a period of time after the rifampin was discontinued. Although the patient was started on ceftriaxone and vancomycin at the same time as rifampin, a literature search failed to identify published reports of drug interactions between tacrolimus and ceftriaxone or vancomycin, which made a drug interaction between rifampin and tacrolimus more likely. In addition, application of the Naranjo nomogram for assessing adverse drug reactions ${ }^{18}$ yielded a score of 5 , which indicated a probable interaction between tacrolimus and rifampin in this patient.

Interaction between tacrolimus and rifampin in adults has been reported previously (Table 1). Five of the 6 published cases involved patients who had undergone renal transplant. ${ }^{12,13,15-17}$ The time between transplant and occurrence of the tacrolimus-rifampin interaction ranged from 4 days to 7 years. As expected, larger baseline doses of tacrolimus were required to meet higher target tacrolimus trough levels in patients whose transplant surgery had occurred more recently. ${ }^{13,16}$ In all but one case, the decrease in tacrolimus trough levels occurred within several days of the patient starting rifampin. The case reported here followed a similar timeline, with the decrease in tacrolimus trough levels observed 4 days after initiation of rifampin. For this patient, an approximately 2 -fold increase in the tacrolimus dose was ultimately required to overcome the induction effects of rifampin. In other published cases, the tacrolimus dose increases ranged from 2-fold to 12-fold (Table 1).

Notably, in some cases, therapeutic tacrolimus trough levels could not be achieved through dose increases alone. ${ }^{15,16}$ López-Montes and others ${ }^{16}$ described a nearly 4 -fold increase in the tacrolimus dose following initiation of antituberculosis treatment with rifampin in a Hispanic patient who had undergone renal transplant. Despite the increased dosage, tacrolimus trough levels remained subtherapeutic (3.7-5.5 ng/mL; target range $10-15 \mathrm{ng} / \mathrm{mL}$ ). The ensuing risk for acute rejection prompted a change in therapy from rifampin to rifabutin. Therapeutic tacrolimus levels were achieved shortly after the switch. Moreno and others ${ }^{15}$ described a similar case of subtherapeutic tacrolimus levels despite a 2-fold dose increase following initiation of rifampin treatment. Therapeutic tacrolimus levels were achieved 6 days after the rifampin was discontinued and replaced with pyrazinamide. Mori and others ${ }^{14}$ also failed to achieve a detectable serum level of tacrolimus following initiation of rifampin therapy in an Asian patient who had undergone bone marrow transplant, despite doubling

Table 1. Characteristics of Reported Cases of Rifampin-Tacrolimus Interactions in Adults

\begin{tabular}{|c|c|c|c|c|c|c|}
\hline \multirow[b]{2}{*}{ Reference (Year) } & \multirow[b]{2}{*}{ Ethnicity } & \multirow[b]{2}{*}{$\begin{array}{l}\text { Target Tacrolimus } \\
\text { Trough (ng/mL) }\end{array}$} & \multicolumn{2}{|c|}{ Tacrolimus Dose (mg/day*) } & \multirow[b]{2}{*}{$\begin{array}{l}\text { Rifampin Dose } \\
\text { (mg/day) }\end{array}$} & \multirow[b]{2}{*}{$\begin{array}{l}\text { CYP3A4 } \\
\text { Inhibitors }\end{array}$} \\
\hline & & & Baseline & $\begin{array}{l}\text { Maximum } \\
\text { (Increase) }\end{array}$ & & \\
\hline Moreno et al. ${ }^{15}$ & Hispanic & $5-10$ & $\begin{array}{c}0.05 \mathrm{mg} / \mathrm{kg} \\
\text { per day }\end{array}$ & $\begin{array}{c}0.1 \mathrm{mg} / \mathrm{kg} \text { per day } \\
\text { (2-fold) }\end{array}$ & Unknown & None \\
\hline $\begin{array}{l}\text { López-Montes } \\
\text { et al. }{ }^{16}\end{array}$ & Hispanic & $10-15$ & 16 & 60 (3.75-fold) & 600 & None \\
\hline Chenhsu et al. ${ }^{12}$ & Asian & $5-8$ & 2 & 24 (12-fold) & 600 & None \\
\hline $\begin{array}{l}\text { Bhaloo and } \\
\text { Prasad }^{13}\end{array}$ & Asian & $10-15$ & 6 & 32 (5.33-fold) & 600 & $\begin{array}{c}\text { Clarithromycin, } \\
\text { diltiazem, } \\
\text { fluconazole }\end{array}$ \\
\hline Mori et al. ${ }^{14}$ & Asian & $5-10$ & 3 & 6 (2-fold) & 300 & Itraconazole \\
\hline $\begin{array}{l}\text { Abdel Halim } \\
\text { et al. }{ }^{17}\end{array}$ & Kuwaiti & $5-7$ & 7 & NAt & Unknown & None \\
\hline This case & White & $5-8$ & 4 & 9 (2.25-fold) & 600 & None \\
\hline
\end{tabular}

NA = not applicable.

*Except where indicated otherwise.

tDose was decreased to $0.5 \mathrm{mg}$ every other day following onset of chronic diarrhea. 
of the tacrolimus dose. Target tacrolimus levels of $5-10 \mathrm{ng} / \mathrm{mL}$ were not reached until the potent CYP3A4 inhibitor itraconazole was added to the patient's regimen.

In contrast to the cases described above, we were able to maintain therapeutic tacrolimus levels of $5-8 \mathrm{ng} / \mathrm{mL}$ during concurrent rifampin therapy by increasing our patient's tacrolimus dose from 4 to $9 \mathrm{mg} /$ day. Nephrotoxicity did not occur with the increasing tacrolimus dose, as evidenced by serum creatinine values that did not exceed baseline levels (Figure 2). To our knowledge, this is the only published case report describing sustained therapeutic tacrolimus levels during rifampin therapy without the addition of CYP3A4 inhibitors or discontinuation of rifampin.

Multiple factors have been reported to influence the pharmacokinetics of tacrolimus, including graft type (e.g., kidney, liver, heart), hepatic and renal function, use of concomitant medications such as corticosteroids, time since transplant, patient's age and ethnic background, hematocrit and albumin concentrations, food intake, diarrhea, and levels of CYP3A and P-glycoprotein expression. ${ }^{19}$ Our patient's hepatic and renal function remained unchanged from baseline throughout her course in hospital. Apart from rifampin, the patient was not started on any medications known to interact with tacrolimus. Both hematocrit and albumin concentrations remained within normal limits and were consistent with baseline values. No episodes of diarrhea were reported during the timeframe of the case report. Of note, the patient's C-reactive protein was elevated at the time of admission, which was likely an indicator of pro-inflammatory cytokines arising from the active bacterial infection. Pro-inflammatory cytokines are known to cause significant reductions in hepatic drug clearance, mostly via decreased production of CYP 450 enzymes. ${ }^{20}$ This phenomenon may explain, in part, why this patient was able to sustain therapeutic tacrolimus levels while taking rifampin.

The observed clinical variability among previous case reports may also be hypothetically attributed to pharmacogenetics. The influence of polymorphisms involving CYP3A4 and P-glycoprotein $(\mathrm{ABCB} 1)$ on tacrolimus pharmacokinetics remains uncertain; however, the influence of CYP3A5 polymorphisms on the pharmacokinetics of tacrolimus is well established. CYP $3 A 5^{*} 1$ allele carriers ("high expressors") are known to require larger doses of tacrolimus to reach target blood concentrations than homozygous carriers of the CYP $3 A 5^{*} 3$ allele ("low expressors"). ${ }^{3-6}$ In addition, CYP3A5 "high expressors" may require a longer period (up to 2 weeks) to achieve target blood concentrations than CYP3A5 "low expressors". ${ }^{4}$ Macphee and others ${ }^{4}$ reported lower tacrolimus concentrations in CYP3A5 "high expressors" during the first 2 weeks after renal transplant, despite therapeutic drug monitoring, and a significant delay in the time to reach target tacrolimus concentrations relative to CYP3A5 "low expressors". Approximately 43\%-73\% of Africans and African Americans and $15 \%-35 \%$ of Asians, but only $5 \%-15 \%$ of whites, are carriers of the $C Y P 3 A 5^{*} 1$ allele. ${ }^{7}$ This observation may explain why several of the reported cases involving Asian and Hispanic patients described failure to meet therapeutic tacrolimus levels after initiation of rifampin. The patient described in the current case report was less likely to be a $C Y P 3 A 5^{*} 1$ carrier, given the lower incidence of the polymorphism in whites. A noncarrier of the $C Y P 3 A 5^{*} 1$ allele would be expected to have lower requirements for tacrolimus and to need a shorter period to achieve steady-state concentrations. This situation might explain why therapeutic tacrolimus levels during rifampin therapy were achieved for the patient described here, whereas addition of CYP3A4 inhibitors or discontinuation of rifampin was required to achieve therapeutic tacrolimus levels in previously reported cases.

Unfortunately, the allele status of our patient and those in previously published cases is unknown. It is unlikely that genotyping of the CYP3A5 enzyme many years after transplant would add any clinical benefit, given the many additional factors that can affect tacrolimus pharmacokinetics. However, it may be beneficial to perform genotyping immediately after transplant surgery, when tacrolimus therapy is being initiated, in order to modify starting doses as appropriate and reach steady state more quickly.

Of note, therapeutic drug monitoring of tacrolimus for the patient in this case was performed by nephrology inpatient clinical pharmacists, who also made dose adjustments of the drug in collaboration with a nephrologist. It is unknown whether the patients described in previously published case reports received similar care. Pharmacists have been recognized as an essential part of the transplant team. Chisholm and others ${ }^{21}$ observed that patients who received clinical pharmacy services were more likely than the control group to reach target immunosuppressant drug levels ( $64 \%$ versus $48 \%, p<0.05$ ). An understanding of the linear pharmacokinetics of tacrolimus, as well as an awareness of the multiple factors that may affect pharmacokinetics, is essential to ensure appropriate dose adjustments. In this case, involvement of a clinical pharmacist familiar with tacrolimus pharmacokinetics ensured that appropriate dose modifications were made and likely contributed to achievement of target tacrolimus levels.

\section{CONCLUSIONS}

To our knowledge, this is the first time a significant drug interaction between rifampin and tacrolimus has been reported in an adult white person. Health care professionals monitoring tacrolimus blood concentrations should be aware of the variability in clinical presentation among reported cases and realize the potential dramatic and sustained effect of this 
drug-drug interaction. This case report, coupled with previously published reports of tacrolimus-rifampin interactions, serves as a reminder of the potent induction effects of rifampin. Involvement of a clinical pharmacist in therapeutic drug monitoring of tacrolimus is beneficial for managing the drug interaction and achieving target drug levels.

\section{References}

1. Staatz CE, Goodman LK, Tett SE. Effect of CYP3A and ABCB1 single nucleotide polymorphisms on the pharmacokinetics and pharmacodynamics of calcineurin inhibitors: part I. Clin Pharmacokinet. 2010;49(3):141-75.

2. Staatz CE, Goodman LK, Tett SE. Effect of CYP3A and ABCB1 single nucleotide polymorphisms on the pharmacokinetics and pharmacodynamics of calcineurin inhibitors: part II. Clin Pharmacokinet. 2010;49(4):207-21.

3. Zhang X, Liu ZH, Zheng JM, Chen ZH, Tang Z, Chen JS, et al. Influence of CYP3A5 and MDR1 polymorphisms on tacrolimus concentration in the early stage after renal transplantation. Clin Transplant. 2005;19(5):638-43.

4. Macphee IA, Fredericks S, Mohamed M, Moreton M, Carter ND, Johnston A, et al. Tacrolimus pharmacogenetics: the $C Y P 3 A 5^{*} 1$ allele predicts low dose-normalized tacrolimus blood concentrations in whites and South Asians. Transplantation. 2005;79(4):499-502.

5. Ferraresso M, Tirelli A, Ghio L, Grillo P, Martina V, Torresani E, et al. Influence of the CYP3A5 genotype on tacrolimus pharmacokinetics and pharmacodynamics in young kidney transplant recipients. Pediatr Transplant. 2007;11(3):296-300.

6. Uesugi M, Masuda S, Katsura T, Oike F, Takada Y, Inui K. Effect of intestinal CYP3A5 on postoperative tacrolimus trough levels in livingdonor liver transplant recipients. Pharmacogenet Genomics. 2006; 16(2):119-27.

7. Lamba JK, Lin YS, Schuetz EG, Thummel KE. Genetic contribution to variable human CYP3A-mediated metabolism. Adv Drug Deliv Rev. 2002;54(10):1271-94.

8. Iwasaki K, Matsuda H, Nagase K, Shiraga T, Tokuma Y, Uchida K. Effects of twenty-three drugs on the metabolism of FK506 by human liver microsomes. Res Commun Chem Pathol Pharmacol. 1993;82(2):209-16.

9. Floyd MD, Gervasini G, Masica AL, Mayo G, George AL Jr, Bhat K, et al. Genotype-phenotype associations for common CYP3A4 and CYP3A5 variants in the basal and induced metabolism of midazolam in Europeanand African-American men and women. Pharmacogenetics. 2003; 13(10):595-606.

10. Burk O, Koch I, Raucy J, Hustert E, Eichelbaum M, Brockmöller J, et al. The induction of cytochrome P450 3A5 (CYP3A5) in the human liver and intestine is mediated by the xenobiotic sensors pregnane $\mathrm{X}$ receptor (PXR) and constitutively activated receptor (CAR). J Biol Chem. 2004; 279(37):38379-85.

11. Kiuchi T, Tanaka K, Inomata Y, Uemoto S, Satomura K, Egawa H, et al. Experience of tacrolimus-based immunosuppression in living-related liver transplantation complicated with graft tuberculosis: interaction with rifampicin and side effects. Transplant Proc. 1996;28(6):3171-2.
12. Chenhsu RY, Loong CC, Chou MH, Lin MF, Yang WC. Renal allograft dysfunction associated with rifampin-tacrolimus interaction. Ann Pharmacother. 2000;34(1):27-31.

13. Bhaloo S, Prasad GV. Severe reduction in tacrolimus levels with rifampin despite multiple cytochrome P450 inhibitors: a case report. Transplant Proc. 2003;35(7):2449-51.

14. Mori T, Aisa Y, Kato J, Nakamura Y, Shimizu T, Okamoto S. Overcoming the effect of rifampin on the tacrolimus metabolism by itraconazole administration in an allogeneic hematopoietic stem cell transplant recipient. Int J Hematol. 2010;91(3):553-4.

15. Moreno M, Latorre A, Manzanares C, Morales E, Herrero JC, Dominguez-Gil B, et al. Clinical management of tacrolimus drug interactions in renal transplant patients. Transplant Proc. 1999;31(6):2252-3.

16. López-Montes A, Gallego E, López E, Pérez J, Lorenzo I, Llamas F, et al. Treatment of tuberculosis with rifabutin in a renal transplant recipient. Am J Kidney Dis. 2004;44(4):e59-63.

17. Abdel Halim M, Al-Otaibi T, Gheith O, El-Kholy O, Abdel Tawab K, Said T, et al. Toxic tacrolimus blood levels with rifampin administration in a renal transplant recipient. Ann Transplant. 2010;15(1):57-60.

18. Naranjo CA, Busto U, Sellers EM, Sandor P, Ruiz I, Roberts EA, et al. A method for estimating the probability of adverse drug reactions. Clin Pharmacol Ther. 1981;30(2):239-45.

19. Staatz CE, Tett SE. Clinical pharmacokinetics and pharmacodynamics of tacrolimus in solid organ transplantation. Clin Pharmacokinet. 2004; 43(10):623-53.

20. Morgan ET. Regulation of cytochromes P450 during inflammation and infection. Drug Metab Rev. 1997;29(4):1129-88.

21. Chisholm MA, Mulloy LL, Jagadeesan M, DiPiro JT. Impact of clinical pharmacy services on renal transplant patients' compliance with immunosuppressive medications. Clin Transplant. 2001;15(5):330-6.

Heather Naylor, BScPharm, ACPR, CDE, is a Clinical Resource Pharmacist, Nephrology, Horizon Health Network, Saint John Regional Hospital, Saint John, New Brunswick.

Jenna Robichaud, BSCPharm, ACPR, is a Clinical Pharmacist, Nephrology, Horizon Health Network, Saint John Regional Hospital, Saint John, New Brunswick.

Competing interests: Heather Naylor received a travel grant from Amgen to attend the 2013 Canadian Society of Nephrology conference. No competing interests declared by Jenna Robichaud.

\section{Address correspondence to:}

Heather Naylor

Saint John Regional Hospital

PO Box 2100

Saint John NB E2L 4L2

e-mail: heather.naylor@horizonNB.ca 